\title{
Electromagnetic radiation by electrons in the corrugated graphene
}

\author{
S. A. Ktitorov ${ }^{1,2}$, R. I. Mukhamadiarov ${ }^{1}$ \\ ${ }^{1}$ Ioffe Institute, St. Petersburg, Russia, \\ ${ }^{2}$ St. Petersburg Electrotechnical University LETI, St. Petersburg, Russia \\ ktitorov@mail.ioffe.ru
}

PACS 78.67.Bf

DOI 10.17586/2220-8054-2016-7-1-51-57

\begin{abstract}
The electromagnetic radiation of electrons in the corrugated graphene has been studied in the presence of a transport electric current in the ballistic regime. We considered here the impact of ripples in monolayer graphene on its electromagnetic properties. Electromagnetic radiation was actually calculated with a use of the standard electromagnetic theory. Two cases: those of regular and random structures were analyzed. The nonlinear relationship between the random height function $h(x, y)$ and the gauge field is shown to create a central radiation frequency distribution peak.
\end{abstract}

Keywords: dipole approximation, graphene, ripples, improper, gauge field.

Received: 20 November 2015

\section{Introduction}

A recent experimental study has shown that the flat geometry of graphene is unstable, leading to the formation of a corrugated structure: topological defects and ripples [1]. Electron motion through the rippled graphene sheet induces electromagnetic radiation. The mechanism for the formation of bremsstrahlung radiation in graphene is similar to that in an undulator or wiggler [2]. While an electron's trajectory in an undulator and wiggler deviates from a direct line due to a periodic system of dipole magnets, the electron's trajectory in graphene becomes curved due to ripples present on the material's surface. The spatial periodicity of the ripples in graphene could reach several hundreds of nanometers [3], which makes the semiclassical approach feasible. Despite the "quasi-relativistic" character of the spectrum, the ratio of Fermi velocity to the speed of light is much smaller, than unity and we can neglect the retardation of electromagnetic radiation. All these points give us a reason to consider the motion of electrons in graphene within the classical approach. The most important mechanisms are bremsstrahlung, cyclotron, and undulator radiation [4]. The emission mechanism under consideration resembles one in the undulator [5] but practically without retardation. We analyzed radiation using a few distinct models. In the first model, we consider a geometric mechanism, directly connected with the presence of undulations, in the second - the pseudo gauge field effect makes the trajectory become curved in the base plane. Both regular and random ripple structures are considered. In the following sections, we will consider various models of electrons motion in corrugated graphene and will derive a formula for the radiation intensity.

\section{Geometric mechanism}

Anomalously high electrons mobility in graphene [4] leads to the mean free path of micron value [6]. For the mean ripples period of 50 nanometers, the ratio of the free path to period of the structure is about 20 , which leads to the $10 \%$ spectral line broadening. The ballistic regime is implemented for the graphene sample of about several microns. Electrons 
motion through the rippled graphene sheet induces an electromagnetic radiation in the terahertz range $[4,7,8]$. The mechanism of formation of the bremsstrahlung radiation in graphene is similar to one in the undulator or wiggler [3]. While the electron trajectory in an undulator and wiggler deviates from the direct line due to the periodic system of the dipole magnets, electron trajectory in graphene is getting curved due to the ripples. A spatial period of ripples in graphene could reach several hundreds of nanometers [1]. This makes the semiclassical approach feasible.

The Fermi velocity vector in the vicinity of the Dirac points in graphene has a constant absolute value of $10^{8} \mathrm{~cm} / \mathrm{s}$. However, its orientation changes over time, leading to a time dependence of the vector components which is responsible for the emission of electromagnetic waves.

The ripple average amplitude is about $1 \mathrm{~nm}$ [9], whereas the period $L \sim 50 \mathrm{~nm}$. Taking this into account, we could assume that velocity preserves a constant value in the direction of the applied field. In other words, electrons in graphene could be considered as an oscillator having the Fermi velocity. The real graphene sample has a random corrugations period that deviates a little from the mean value. The chaotic surface could be considered as a superposition of sinusoids with their own period and height. This approach is widely used for wave analysis in radiophysics. In order to describe the random process of electromagnetic radiation within a limited spectrum, we introduce the random function $h(x)$ that plays a role of the ripples height relative to the base plane. Assuming the inhomogeneities to be one-dimensional and taking into account small ratio of $\frac{h_{m}}{L} \ll 1$ we can write the following equation for the velocity:

$$
v_{x}=v_{F}, \quad v_{z}=v_{F} \frac{d}{d x} h(x(t)) .
$$

That means electromagnetic radiation spreads in a normal direction and infinitesimal changes in the in-plane velocity components are higher order effects [10] and can be neglected. We calculate the magnetic field induced by a moving charge at the deliberately chosen point with use of retarded potentials. The Fourier transform of the vector potential reads:

$$
\mathbf{A}_{\omega}=\frac{e e^{i k r}}{c r} \int_{0}^{\infty} \mathbf{v}(t) e^{i\left(\omega t-\mathbf{k r}_{\mathbf{0}}\right)} d t
$$

where $c$ is the speed of light, $e$ is the electrons charge, $\mathbf{r}$ is the radius vector, $\mathbf{r}_{\mathbf{0}}$ is the moving charge radius vector.

Taking (1) into account and excluding the retardation effect, we have:

$$
A_{z}=\frac{e e^{i k r}}{c r} v_{F} \int_{0}^{\infty} \frac{d}{d x} h(x(t)) e^{i t \omega} d t .
$$

The other two components will either be constant or zero and do not contribute to the generation of electromagnetic waves. The Fourier component of the magnetic field becomes:

$$
B_{y}=-i k_{x} \frac{e e^{i k r}}{c r} v_{F} \int_{0}^{\infty} \frac{d}{d x} h(x(t)) e^{i t \omega} d t .
$$

The magnetic field enters the intensity formula in squared form:

$$
\left|B_{y}\right|^{2}=\left(k_{x} \frac{e}{c r} v_{F}\right)^{2} e^{i k r} e^{-i k r} \int_{0}^{\infty} d t \frac{d}{d x} h(x(t)) e^{i \omega t} \cdot \int_{0}^{\infty} d t^{\prime} \frac{d}{d x^{\prime}} h\left(x^{\prime}\left(t^{\prime}\right)\right) e^{-i \omega t^{\prime}} .
$$


In the case of random surface of the graphene, the squared absolute value can be represented as a multiplication of two equations where distribution of random function $h(x)$ could be considered as realizations, which could differ even in the same point [11]. Therefore, while calculating the field intensity, the relationship between them could be represented in terms of the correlation function. We introduce it by averaging (5) over the ripples' configuration:

$$
\begin{aligned}
& \left\langle\left|B_{x}\right|^{2}\right\rangle=-k_{y}^{2} \frac{e^{2} v_{F}^{2}}{c^{2} r^{2}} \int_{0}^{\infty} d t \int_{0}^{\infty} d t^{\prime} \frac{d^{2}}{d x^{2}}\left\langle h(x(t)) h\left(x^{\prime}\left(t^{\prime}\right)\right)\right\rangle e^{i \omega\left(t-t^{\prime}\right)}, \\
& \left\langle\left|B_{y}\right|^{2}\right\rangle=-k_{x}^{2} \frac{e^{2} v_{F}^{2}}{c^{2} r^{2}} \int_{0}^{\infty} d t \int_{0}^{\infty} d t^{\prime} \frac{d^{2}}{d x^{2}}\left\langle h(x(t)) h\left(x^{\prime}\left(t^{\prime}\right)\right)\right\rangle e^{i \omega\left(t-t^{\prime}\right)},
\end{aligned}
$$

where the angle brackets stand for configuration averaging. The random process $h(x)$ could be considered as Gaussian and stationary with the correlator:

$$
\left\langle h(x) h\left(x^{\prime}\right)\right\rangle=K\left(x-x^{\prime}\right)=K(\xi) .
$$

Here, the correlation function value depends on relative coordinate $x-x^{\prime}$, which means that statistical characteristics are invariant under a shift along the $O X$. Assuming this process to be one with a narrow band spectrum, we write the correlator in the form:

$$
K(\xi)=\left\langle h^{2}\right\rangle e^{-\alpha|\xi|} \cos \gamma \xi,
$$

where $\left\langle h^{2}\right\rangle$ is the dispersion, $\alpha$ is the inverse correlation radius and $\gamma$ is the inverse mean period and $\alpha \ll \gamma$. Making the substitution $\xi=x-x^{\prime}, \tau=t-t^{\prime}, T=\left(t+t^{\prime}\right) / 2$, we can rewrite formula (6) in the form:

$$
\left\langle\left|B_{x}\right|^{2}\right\rangle=\left(\frac{k_{y}^{2} e^{2}}{r^{2}}\right) \frac{v_{F}^{2}}{c^{2}} \frac{1}{\alpha^{2}} \frac{\omega^{2}}{v_{F}^{2}}\left\langle h^{2}\right\rangle \int_{0}^{\infty} d T \int_{0}^{\infty} d \tau e^{-\alpha v_{F}|\tau|} \cos \left(\gamma v_{F} \tau\right) e^{i \omega \tau} e^{i T\left(\omega-\omega^{\prime}\right)} e^{i \tau\left(\omega+\omega^{\prime}\right) / 2}
$$

Integration over the difference variable makes a Fourier transform, whereas integration over sum variable is simply the time required for an electron to travel through graphene sample:

$$
\left\langle\left|B_{x}\right|^{2}\right\rangle=-\delta\left(\omega-\omega^{\prime}\right) \frac{k_{y}^{2} e^{2}\left\langle h^{2}\right\rangle \omega^{2}}{c^{2} r^{2}}\left(\frac{\alpha v_{F}}{\alpha^{2} v_{F}^{2}+\left(\omega+\gamma v_{F}\right)^{2}}+\frac{\alpha v_{F}}{\alpha^{2} v_{F}^{2}+\left(\omega-\gamma v_{F}\right)^{2}}\right) .
$$

Spectral distribution of electromagnetic radiation

$$
\frac{d^{2} \mathscr{E}}{d \omega d o}=\frac{c}{4 \pi^{2}}\left\langle\left|B_{x}\right|^{2}\right\rangle r^{2}
$$

Substituting (11) in (12) we obtain the formula for the spectral intensity of electromagnetic radiation in rippled graphene:

$$
\frac{d^{2} \mathscr{E}}{d \omega d o}=\delta\left(\omega-\omega^{\prime}\right) \frac{k_{y}^{2} e^{2}\left\langle h^{2}\right\rangle \omega^{2}}{4 \pi^{2} c}\left(\frac{\alpha v_{F}}{\alpha^{2} v_{F}^{2}+\left(\omega+\gamma v_{F}\right)^{2}}+\frac{\alpha v_{F}}{\alpha^{2} v_{F}^{2}+\left(\omega-\gamma v_{F}\right)^{2}}\right) .
$$

Integrating (13) by frequencies and angle

$$
P=\frac{c}{4 \pi} \int_{-\infty}^{\infty} d \omega \int_{0}^{2 \pi} d \Omega\left\langle\left|B_{x}\right|^{2}\right\rangle r^{2}
$$


we obtain the relation for electromagnetic radiation power per one electron:

$$
P_{e}=\int_{-\infty}^{\infty} d \omega \int_{0}^{2 \pi} d \Omega \cos \theta \frac{e^{2} \omega^{4}\left\langle h^{2}\right\rangle}{4 \pi c^{3}}\left(\frac{\alpha v_{F}}{\alpha^{2} v_{F}^{2}+\left(\omega+\gamma v_{F}\right)^{2}}+\frac{\alpha v_{F}}{\alpha^{2} v_{F}^{2}+\left(\omega-\gamma v_{F}\right)^{2}}\right)
$$

We see that the majority of the electromagnetic radiation comes with a frequency corresponding to the mean period of the ripples structure with some broadening due to its random character. The term in brackets yields the Lorentz distribution.

In the limit of $\alpha \rightarrow 0$ the chaotic surface becomes a regular sine and we obtain the formula:

$$
P_{e}=\int_{-\infty}^{\infty} d \omega \int_{0}^{2 \pi} d \Omega \cos \theta \frac{e^{2} \omega^{4} h_{m}^{2}}{4 c^{3}} \delta\left(\omega-\gamma v_{F}\right)=\frac{\pi e^{2}\left(\gamma v_{F}\right)^{4} h_{m}^{2}}{c^{3}}
$$

The obtained formulae establish a relationship between the radiation spectral density $\frac{d P}{d \omega}$ and the correlator of ripples. This can be used to investigate of the ripples' morphologies.

For ripples period $L=50 \mathrm{~nm}$, maximum amplitude $h_{m}=1 \mathrm{~nm}$ and area of graphene membrane $S=10^{-4} \mathrm{~cm}^{2}$, we obtain power of electromagnetic radiation of order several $\mathrm{mW}$.

\section{Gauge mechanism}

In this part of our work, we consider the synthetic gauge field effect on electromagnetic wave emission. The nature of these fields is following. Electronic states in flat graphene can be written by means of the tight binding model equations, which due to linear spectra in Dirac point vicinity take form of Dirac - Weyl equations. The graphene membrane bending modifies the electronic states spectra. Moreover, this influence can be represented by introducing vector potential, rotor of which people called synthetic magnetic field [1]. In fact, these "fields" create new energy levels, similar to Landau levels, and mimics Ahoronov - Bohm effect [1]. The elasticity theory gives the following relationships between the out-of-plane displacement $h(y, z)$ and the gauge field vector potential components [12]:

$$
A_{y}=-\frac{\beta}{a} \frac{d h}{d x} \frac{d h}{d y}, \quad A_{x}=\frac{1}{2} \frac{\beta}{a}\left(\left(\frac{d h}{d x}\right)^{2}-\left(\frac{d h}{d y}\right)^{2}\right), \quad \mathcal{H}_{z}=\frac{\partial A_{y}}{\partial x}-\frac{\partial A_{x}}{\partial y} .
$$

Here, $a$ is the lattice constant and $\beta$ is a dimensionless parameter. It now becomes apparent that gauge fields in graphene occur only when the structure is inhomogeneous in both in-plane directions. In other words, graphene sheet has to have "humps and hollows" in its structure.

Here, we consider the case of regular undulation in both directions:

$$
h(x, y)=h_{m} \sin \gamma x \sin \gamma y .
$$
field:

Substitution of $h(x, y)$ from (18) into (17) gives an expression for the synthetic magnetic

where the amplitude is determined as follows:

$$
\mathcal{H}_{z}=\mathcal{H}_{0} \sin (2 y \gamma)(-2+\cos (2 x \gamma))
$$

$$
\mathcal{H}_{0}=\frac{h_{m}^{2} \gamma^{3}}{2 a} .
$$

The synthetic field $\mathcal{H}$ can be expressed in terms of the "real" field $H$ in the following manner:

$$
H=\frac{c \hbar}{e} \mathcal{H}
$$


After this link has been made, we can modify the equations from [11] and define the undulator-like trajectory for "massless" electrons in graphene:

$$
\begin{aligned}
\frac{\mathcal{E}}{v_{F}^{2}} \frac{d^{2} x}{d t^{2}} & =-e \frac{d y}{d t} H \cos \gamma x, \\
\frac{\mathcal{E}}{v_{F}^{2}} \frac{d^{2} y}{d t^{2}} & =e \frac{d x}{d t} H \cos \gamma x,
\end{aligned}
$$

where $\mathcal{E}$ is the electron energy. The solution reads:

$$
\cos \gamma x=\cosh \gamma y-\frac{1}{k} \sinh \gamma y \text {. }
$$

The resulting trajectory differs slightly from a simple sine function. Assuming that $y \gamma \ll 1$, we can simplify the last formula:

$$
y=y_{m} \sin ^{2} \frac{\gamma x}{2}
$$

where the deviation amplitude is:

$$
y_{m}=\frac{2 k}{\gamma}=\frac{2 v_{F} \hbar h_{m}^{2} \gamma}{a \mathcal{E}} .
$$

The estimations show the maximum amplitude of in-plane deviations to be much smaller than the mean period for the ripples and have values similar to ripples' amplitudes. This makes us think that electrons actually move along complex, helix-like trajectory.

These formulae show that gauge fields in graphene have a real influence on an electron's trajectory and induce in-plane oscillations. The magnitude of the effect depends upon sample parameters and free electron energy.

Now, with (22), we are able to derive the formula for power. Substituting the expression for an oscillating electron's velocity $v_{y}=v_{F} \dot{y}$ into (17), we obtain the formula for the radiation vector potential:

$$
A_{y}=\frac{e e^{i k r}}{c r} \int\left(y_{m} \gamma\right) v_{F} \sin \left(\gamma v_{F} t\right) e^{i \omega t} .
$$

Thus, we have the expression for the radiation power spectral density for the case of harrmonic ripple structure:

$$
P(\omega, \theta)=\frac{1}{4 \pi} \int d \omega \int d \theta \frac{e^{2} v_{F}^{2} \omega^{2}}{2 c^{3}}\left(y_{m}^{2} \gamma^{2}\right) \cos \theta\left(\delta\left(\omega+\gamma v_{F}\right)+\delta\left(\omega-\gamma v_{F}\right)\right) .
$$

Finally, integrating the expression and substituting for $y_{m}$, we derive the formula for the electromagnetic radiation power in terms of the sample parameters:

$$
P=2 \pi^{2} \frac{e^{2} v_{F}^{4}}{c^{3}} \frac{h_{m}^{2}}{L^{4}}\left(\left(\frac{v_{F} \hbar / a}{\mathcal{E}}\right)\left(h_{m} \gamma\right)\right)^{2} .
$$

To understand the role of gauge fields in whole picture of the radiation process, it will be useful to compare power formula obtained in geometric model with (28)

$$
\frac{P_{\text {gauge }}}{P_{\text {geom }}}=\frac{1}{8 \pi^{3}}\left(\frac{v_{F} \hbar}{a \mathcal{E}} \frac{h_{m}}{L}\right)^{2} .
$$

This ratio would be of order of unity for the same parameters of graphene structure at $\varepsilon=0.1 \mathrm{eV}$ energies.

Calculations for the case of the random function $h(x, y)$ are carried out similarly to the case of the geometric mechanism but with one important distinction: a nonlinear relation (17) between the narrow band spectrum random function $h(x, y)$ and the gauge field $A$ induces the 
appearance of a central radiation peak of. Really, let us determine the quadratic relation between the random functions:

$$
g(t)=(h(t))
$$

When $g(h)=a h^{2}$, the correlator $\langle g(t) g(0)\rangle$ can be expressed as follows [11]:

$$
\langle g(t) g(0)\rangle=a^{2} \sigma^{4}\left[1+2\langle h(t) h(0)\rangle^{2}\right],
$$

where $\sigma$ is the $h(t)$ process variance $\sigma=\left\langle h^{2}\right\rangle$.

We do not aware about existing experimental data or theoretical results regarding actual correlation function's $\left\langle h(x) h\left(x^{\prime}\right)\right\rangle$ form. All we can do is estimate dispersion and correlation radius. A reasonable choice would be the simplest form that does not lead to any disagreement. We choose the exponential decay for geometry model, i.e. Lorentz-shaped energy spectra. In case of gauge fields, the exponential correlator yields singularity: it corresponds to infinitely large gradients of random function [14]. Thus, we choose the Gaussian correlator form:

$$
K(x)=\frac{\left\langle h^{2}\right\rangle \alpha^{3}}{8 \pi \sqrt{\pi}} e^{-x^{2} \alpha^{2} / 4} \cos (\gamma x) .
$$

Substituting (32) in (31) we have

$$
\langle g(t) g(0)\rangle=b^{2} \sigma^{4}\left[1+2\left(\frac{\left\langle h^{2}\right\rangle \alpha^{3}}{8 \pi \sqrt{\pi}}\right)^{2} e^{-2\left(v_{F} t\right)^{2} \alpha^{2} / 4} \cos ^{2}\left(\gamma v_{F} t\right)\right] .
$$

Taking into account formula (17), we obtain for the gauge field random process:

$$
\begin{aligned}
& \int_{-\infty}^{\infty} d x e^{i k x} K^{2}(x)= \\
& \quad b^{2} \sigma^{4}\left(\frac{\left\langle h^{2}\right\rangle \alpha^{3}}{8 \pi \sqrt{\pi}}\right)^{2} \int_{0}^{\infty} d x e^{-\left(\alpha^{2} x^{2} / 2\right)}[\cos (k x)+[\cos (k+2 \gamma) x+\cos (k-2 \gamma) x]] .
\end{aligned}
$$

Carrying out the integration, we obtain the spectrum comprising the central and highfrequency components:

$$
P \propto P_{0}+b^{2} \sigma^{4} \frac{\left\langle h^{2}\right\rangle^{2} \alpha^{5} k^{3}}{16 \pi^{2} \sqrt{\pi}}\left[e^{-k^{2} / \alpha^{2}}+\left[e^{-(k+2 \gamma)^{2} / \alpha^{2}}+e^{-(k-2 \gamma)^{2} / \alpha^{2}}\right]\right] .
$$

The pre-exponent term $k^{3}$ in (35) accounts for differentiation in (17) formula. On the Fig. 1 we presented the spectral distribution of a radiation power, divided by $k^{3}$.

Thus, the quadratic relation between velocity and the Monge variable leads to a rise of the electromagnetic radiation central peak.

\section{Conclusion}

We have considered here the impact of ripples in monolayer graphene on its electromagnetic properties. Two mechanisms of undulator-like radiation are considered: a geometric mechanism, directly connected with the presence of undulations; and the pseudo gauge field effect, which makes the trajectory become curved in the base plane. The electromagnetic radiation was actually calculated using the standard retarded potential. For both of mechanisms, two cases of regular and random structures are analyzed. The nonlinear relation between the random height function $h(x, y)$ and the gauge field $A$ is shown to create a central radiation frequency distribution peak. Our results can be used for study of ripples morphology and for generation of terahertz radiation. 


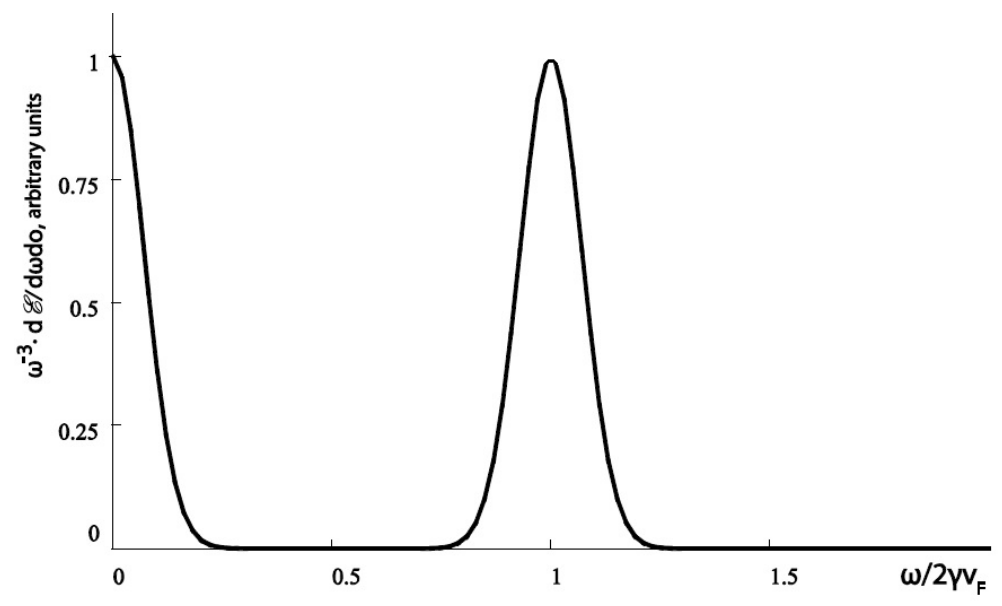

FIG. 1. Central and side radiation peaks in gauge model

\section{References}

[1] Vozmediano M.A.H., Katsnelson M.I., Guinea F. Gauge fields in graphene. Physics Reports, 2010, 496, P. 109-148.

[2] Alferov D.F., Bashmakov Yu.A., Cherenkov P.A. Radiation from relativistic electrons in a magnetic undulator. Sov. Phys. Usp., 1989, 32 (3), P. 200-227.

[3] Castro Neto A.H., Guinea F., et al. The electronic properties of grapheme. Rev. Mod. Phys., 2009, 81, P. 109-162.

[4] Tantiwanichapan K., DiMaria J., Melo S.N. Graphene electronics for terahertz electron-beam radiation. Nanotechnology, 2013, 24, P. 375205-375212.

[5] Motz H. Applications of the Radiation from Fast Electron Beams. J. Appl. Phys., 1951, 22, P. 527-535.

[6] Mayorov A.S., Gorbachev R.V., et al. Micrometer-scale ballistic transport in encapsulated graphene at room temperature. arxiv:1103.4510, 2011.

[7] Zhan T., Han D., et al. Tunable terahertz radiation from graphene induced by moving electrons. Phys. Rev. B, 2014, 89, 245434 (1-7).

[8] Llatser I., Kremers C., et al., Radiation Characteristics of Tunable Graphennas in the Terahertz Band. Phot. Nanostr., 2012, 21, P. 946-952.

[9] Castro Neto A.H., Guinea F., et al. The electronic properties of graphene. Rev. of Modern Phys., 2009, 81, P. 109-162.

[10] Zulicke U., Winkler R., Bolte J. Nanospintronics meets relativistic quantum physics: Ubiquity of Zitterbewegung effects. Physica E, 2008, 40, P. 1434-1435.

[11] Stratonovich R.L. Topics in the theory of random noise. Gordon and Breach Science Publishers, New York - London, 1963, 216 p.

[12] Guinea F., Baruch H., Le Doussal P. Gauge field induced by ripples in graphene. Phys. Rev. B, 2008, 77, $205421(1-8)$.

[13] Combe R., Feix M. Mouvement dun electron dans un ondulateur magnetique. Comt. Rend., 1953, 237, P. 1318-1320.

[14] Chernov L.A. The waves in randomly inhomogeneous media. Science, Moscow, 1975. 